\title{
LA MÉDIATION CULTURELLE DANS LES MUSÉES : UNE FORME DE RÉGULATION SOCIALE
}

\author{
Daniel Jacobi, Anik Meunier et Sylvie Romano ${ }^{1}$
}

\section{Introduction}

Depuis quelques années, la fréquentation des équipements muséographiques a augmenté, parfois de façon spectaculaire. On sait que cette croissance de la fréquentation est le résultat de deux tendances : d'une part, la multiplication des visites d'expositions par les amateurs cultivés et, d'autre part, la conquête de nouvelles catégories de visiteurs. Non seulement, le public dans les musées est plus nombreux, mais il s'est diversifié, devenant plus hétérogène. Pour faire davantage d'entrées et atteindre ses objectifs de fréquentation, un équipement muséographique doit, en premier lieu faire le plein des visiteurs captifs (pour l'essentiel, constitué de publics scolaires), et en second lieu, faire venir, aussi bien les touristes lointains (parfois en prospectant les organisateurs de voyages), que les proches habitants et les familles en promenade, tout comme, en morte saison, les groupes de retraités. Tout cela,

1 Université de Bourgogne.

Recherches en communication, $\mathrm{n}^{\circ} 13,(2000)$. 
évidemment, sans oublier de fidéliser les visiteurs passionnés et les faire revenir à l'occasion des expositions temporaires... ${ }^{1}$

Pour accompagner cette politique d'accueil et de diversification des publics, des services spécifiquement conçus pour mieux répondre à leurs besoins ont été créés. Au traditionnel bureau éducatif ou pédagogique confié à un enseignant et voué à l'accueil des groupes scolaires, ont fait place dans l'organigramme des services plus étoffés. Qu'ils se nomment service culturel ou accueil des publics, ils ont en charge d'acccueillir les différentes catégories de visiteurs et de satisfaire leurs attentes. Il ne s'agit plus seulement de mettre en place une offre éducative qui tente de répondre à des exigences précises, mais aussi d'offrir toute une panoplie de prestations tout en procurant des conditions de visite adaptées au plus grand nombre de visiteurs. De la visite guidée classique conduite par un conférencier, à la réponse originale et individualisée à des demandes de visiteurs exigeants, on assiste à une mutation et une diversification des formes d'intervention et de présence auprès du public, toutes interventions et prestations que l'on regroupe sous le terme vague mais commode de médiation $^{2}$

Il faut cependant reconnaître que la place davantage visible de la médiation dans l'institution ne fait que poursuivre et faire apparaître au grand jour l'une des missions attribuées au musée ${ }^{3}$. La conservation des œuvres et la recherche sur celles-ci, l'aménagement d'une collection comme l'organisation d'expositions temporaires vont de pair avec la mission d'acculturation qui dans notre société est dévolue aux musées. À la conception de seules activités pédagogiques qui condensaient cette mission d'éducation ont fait place une réflexion plus. diffuse sur le choix des thèmes, des contenus artistiques, thématiques ou scientifiques et surtout sur la façon de les divulguer en se préoccupant davantage des caractéristiques et de la nature des publics à qui ils sont destinés.

Ceci a eu pour effet de modifier les stratégies et les moyens autrefois mis en œuvre par les professionnels de la médiation comme le révèle les deux enquêtes sur lesquelles ce texte prend appui. La

1 J. Eidelman, "Qui fréquente les musées à Paris ?", Publics et Musées, n², 1992, pp. 19-47.

2 E. CAILLET, A l'approche du musée, la médiation culturelle, Lyon, PUL, coll. "Muséologie", 1995.

3 D. Poulot, "Bilan et perspectives pour une histoire naturelle des musées", Publics et Mudées, $\mathrm{n}^{\circ} 2$ 2, 1992, pp. 125-147. 
première est une étude qualitative qui a été conduite auprès d'un groupe de professionnels de la médiation culturelle. L'objectif de l'enquête a été de comprendre et décrire un phénomène dans toute sa complexité : comment les formes de la médiation culturelle dans les musées régulent-elles ou conditionnent-elles les comportements des visiteurs? Le corpus consiste en une quinzaine d'entretiens approfondis à questions ouvertes, administrées aux professionnels de la médiation ayant à conduire des tâches de prise en charge des visiteurs. Pour témoigner de la diversité des pratiques, ces acteurs de la médiation ont été sélectionnés dans différents types de musées : beaux-arts, art contemporain, sciences \& techniques, histoire \& société1.

La seconde recherche porte sur l'analyse détaillée d'une activité de médiation très structurée organisée en dehors de toute référence aux contenus scolaires pour des jeunes enfants non lecteurs (4 à 6 ans). Cette activité a été observée, décrite et analysée. Puis, elle a été évaluée à l'aide d'une méthode originale à l'issue de l'activité, ainsi qu'une semaine et un mois après celle-ci² ${ }^{2}$. Ici encore, nous essaierons de percevoir la nature des effets de la médiation culturelle sur les comportements de visite.

\section{Formes et enjeux de la médiation dans les musées}

Longtemps les musées n'ont été fréquentés que par un public cultivé de connaisseurs venus pour se délecter, se cultiver ou parfaire ses connaissances. Pourtant, les choses changent s'ils accueillent maintenant d'autres publics en dehors des adultes bien éduqués, comme par exemple des groupes sociaux handicapés, exclus et peu formés ${ }^{3}$. Les besoins des plus jeunes sont différents de ceux des aînés et les attentes des plus démunis sont bien divergentes de celles des plus cultivés. Toutefois, on aurait tort de considérer que les besoins des plus démunis constituent le socle de la médiation. Il n'est pas étonnant de compter les enseignants parmi les visiteurs du week-end

1 A. MEunier, Les acteurs de l'éducation dans le musée : analyse d'entretiens centrés sur leurs pratiques professionnelles, Mémoire de DEA Muséologie; Publics, Expositions, Institutions, Dijon, Université de Bourgogne, 1996.

2 S. Romano, Le musée a-t-il un impact pédagogique sur les enfants de 4 à 6 ans?, Mémoire de DEA Muséologie ; Publics, Expositions, Institutions, Dijon, Université de Bourgogne, 1996.

3 O. COPPEY, "Le musée non-lieu de formation, Media Scope, $\mathrm{n}^{\circ} 3$, Versailles, CRDP, 1992, pp. 75-78. 
ainsi que des adultes cultivés et experts en une discipline spécifique venus accompagnés de leurs enfants. C'est pourquoi, pour garder leur prestige et leur rayonnement, les institutions muséographiques doivent continuer à apparaître comme d'irremplaçables centres de ressources capables de satisfaire des petits groupes comme les enseignants, les critiques et les experts. Ce sont ces catégories stratégiques qui relayeront le travail du musée et fixeront sa réputation de médiation.

Pourtant, il ne faut pas perdre de vue que les visiteurs des expositions, toutes natures confondues, ne sont pas dans leur majorité des spécialistes des thèmes exposés. Et, compte tenu de l'inscription de cette pratique pendant la période des loisirs, l'activité de visite doit satisfaire à des conditions minimales pour avoir quelque chance d'être perçue favorablement : qu'elle conserve un caractère de convivialité comparativement à d'autres médias, qu'elle suscite de l'étonnement, qu'elle éveille la curiosité et surtout qu'elle procure du plaisir...

\subsection{Optimiser la communication culturelle auprès d'un large public}

C'est la présence de groupes de visiteurs peu familiers avec les thèmes présentés dans les musées qui conduit l'équipe de médiation à concevoir et proposer des programmes et des dispositifs destinés à soutenir et à aider les visiteurs des expositions (feuillets pédagogiques, conférences, visites...). On cherche à faire découvrir et aimer les activités proposées en éveillant la curiosité, en stimulant l'intelligence, en faisant appel à l'émotion, en favorisant l'expression et les échanges entre les visiteurs. L'objectif est d'offrir un moment enrichissant autant qu'agréable, qui donne envie de revenir pour poursuivre, à son rythme, l'exploration amorcée parfois lors d'une sortie dans un cadre scolaire ${ }^{1}$.

Conscients des disparités entre les diverses catégories de publics (scolaires ou autres), les musées mettent en œuvre un éventail de programmes adaptés à leurs différentes catégories de visiteurs. Les formes des activités de médiation se divisent d'abord en deux grandes catégories : d'un côté, les scolaires et les visiteurs captifs venus en groupes organisés (retraités, voyages touristiques, comités

1 L. DIERKING et J. FAlK, The Museum Experience, Washington, Whalesback Books, 1992. 
d'entreprise...) et, de l'autre, les visiteurs spontanés individuels ou en petits groupes (adultes, familles...).

Les activités destinées aux groupes scolaires rejoignent ce qu'il est convenu d'appeler l'éducation non formelle, extra-scolaire ou périscolaire. Elles visent à initier, compléter, voire même prolonger les enseignements scientifiques ou artistiques dispensés dans le cadre scolaire'. Ainsi, il n'est pas rare de constater que la plupart des activités proposées aux groupes en provenance des établissements scolaires rejoignent les objectifs des programmes d'étude ${ }^{2}$. Par contre, les activités qui s'adressent aux autres publics, notamment les adultes, tentent de s'affranchir de la perspective de continuité et de prolongement de la formation entamée dans un cadre scolaire ou institutionnel.

Quoi qu'il en soit, la panoplie des formes d'intervention de la médiation culturelle est bien plus étendue qu'on ne l'imagine parfois. Outre les choix didactiques intégrés dans la conception des expositions elles-mêmes, de nombreux moyens parallèles ont été expérimentés : mise en place de salles de découvertes pour les groupes scolaires ou les jeunes visiteurs venus avec leurs parents, intégration d'aides à l'interprétation dans l'exposition, démonstrations d'expériences scientifiques, projections audiovisuelles ou vidéo, expériences et travaux pratiques dans des laboratoires, ateliers artistiques pour les aînés... Bref, des traditionnelles visites commentées pour les adultes ou les enfants, aux projets de théâtre pour les adolescents, il existe toute une panoplie de moyens plus ou moins inventifs mis en place dans les différents musées.

\subsection{Trois types de médiation}

En généralisant, on peut distinguer trois grandes formes de médiation : la conquête de nouveaux visiteurs, la communication dans (et autour) de l'exposition, la création d'actions spécifiques. Sans dresser un catalogue exhaustif de toutes ces actions de médiation, il est nécessaire de distinguer d'abord ce qui relève de l'incitation à

1 M. Allard et C. Gauthier, "Le musée dans les programmes scolaires (18611989)", Traces, vol. $28, \mathrm{n}^{\circ} 2$, pp. 34-35.

2 J. EIDELman et J. Peignoux, "Les enseignants de l'école primaire et la Cité des Sciences : les conditions du partenariat école-musée", La lettre de l'OCIM, $\mathrm{n}^{\circ} 37$, Dijon, OCIM, 1995, pp. 17-25. 
venir (politiques de sensibilisation conduites en direction des différentes catégories de publics comme les étudiants, les jeunes des banlieues, les comités d'entreprise, l'accueil des groupes de visiteurs, la formation de personnes relais animant des groupes de visites...).

Il est par contre plus difficile d'identifier la place de la médiation dans les expositions : les affiches et la communication à propos des nouvelles expositions, la conception de publications autres que le catalogue, l'organisation physique et matérielle des expositions dans le musée et de la signalétique, le mode de rédaction des étiquettes, etc. sont en soi des aides à l'interprétation qui relèvent autant du travail des concepteurs des expositions que de celui des médiateurs ${ }^{1}$. Enfin, il est plus aisé de repérer les actions de médiation proprement dites: fabrication et mise en place d'aides à l'interprétation (fiches, livrets, feuillets d'aide à la visite), formation des guides-animateurs de visites, conception de programmes et d'activités culturelles de découverte des collections, journées exceptionnelles portes ouvertes, etc.

En outre, dans certains équipements, des spécialistes de la médiation ou de l'éducation conçoivent ou font réaliser des outils spécifiques : petites expositions pédagogiques, feuillets didactiques, panneaux d'interprétation, textes destinés aux audioguides, cahiers pédagogiques, périodiques, livres pour enfants, scénarios de vidéos, de diaporamas, et autres dispositifs interactifs. De plus, ils conçoivent et expérimentent les mallettes, meubles ou dispositifs pédagogiques, les programmes de visites par niveau d'études... comme ils participent à l'aménagement des espaces éducatifs dans l'exposition ou des salles de découvertes.

\subsection{La médiation dirigée vers les enfants non lecteurs}

Parmi les différents publics vers lesquels les nouvelles formes de médiation des musées se sont orientées, nous nous attarderons sur le cas de tout jeunes enfants. Il faut signaler que des équipements muséographiques ont même été spécialement conçus pour eux : le Musée en Herbe à Paris, la Cité des enfants de la Villette à Paris, le Préau des Accoules à Marseille, et à l'étranger, le Musée pour les

1 D. JACOBI et A. MeUnier, “Au service du projet éducatif de l'exposition : l'interprétation", La lettre de l'OCIM, Dijon, OCIM, 1999. 
enfants de Boston, le musée de la science pour les enfants de Caracas, le Musée pour enfants de Bruxelles...

Le Préau des Accoules, qui a servi de cadre à cette étude, est un édifice monumental, construit entre le $\mathrm{XVII}^{\mathrm{e}}$ et le $\mathrm{XVIII}^{\mathrm{e}}$ siècle, transformé depuis 1991 en un lieu dédié à la sensibilisation des enfants aux arts et au patrimoine. Pour cela, et malgré le fait qu'il ne possède aucune collection, il organise chaque année trois expositions/animations thématiques pour le jeune public dont les seuls titres sont évocateurs : les Indiens, l'Afrique, la mer... Grâce à ces événements, les enfants peuvent, souvent pour la première fois, visiter une exposition et être en contact avec des œuvres authentiques qu'ils apprennent à connaître et à estimer par le biais de l'animation proposée.

Parmi les différentes médiations que le Préau des Accoules a présenté en 1997/1998, c'est une exposition de peinture Camoin au balcon un peintre et son monde qui a fait l'objet de la recherche citée plus haut. Cette exposition était destinée à des enfants de quatre à onze ans. Pour des raisons empiriques, on a observé exclusivement des groupes d'enfants appartenant à des catégories très voisines : ils sont tous non lecteurs ou mauvais lecteurs (classes de grande section des écoles maternelles, cours préparatoire des écoles primaires, enfants les plus jeunes des centres de loisirs).

\section{Comment mesurer les résultats ou les effets de la médiation dans les musées}

Il est évident que le but de toutes ces actions de médiation est de créer des conditions favorables à une appropriation de l'exposition et de ses contenus par tous les visiteurs ; autrement dit, le but est de favoriser le fonctionnement de la communication culturelle et au-delà générer l'acculturation. En comparant leurs différentes formes entre elles, il est possible en première approximation de se faire une idée des qualités des dispositifs d'aide à la visite ou d'aide à l'interprétation. Si certains demeurent abscons et illisibles, parce que rédigés par des spécialistes dans leur jargon ${ }^{1}$, les partis pris pédagogiques retenus sont nettement visibles dans beaucoup de

1 D. JACOBI, "Des formes simples pour identifier et interpréter les objets dans les expositions", in F. LAPEYRE (édit.), Simple-simplification, Les cahiers du français contemporain, $\mathrm{n}^{\circ}$ 1, CREDI-Didier érudition, 1994, pp. 195-212. 
documents édités : des notices explicatives, des commentaires oralisés, des guides, des parcours thématiques, des panneaux d'interprétation, des plans d'orientation, des jeux de piste, des livretsjeux, des jeux de rôles... Ces dispositifs, qui sont, soit proposés aux visiteurs eux-mêmes, soit fournis aux enseignants ou aux accompagnateurs peuvent se substituer à la présence d'un médiateur au sein de l'exposition.

Néanmoins, il faut souligner que l'éducation muséale et la médiation, aussi novateurs qu'ils soient, ont rarement fait l'objet d'évaluations instrumentées. Dans un secteur qui est longtemps resté à l'écart de toute innovation en matière d'accueil de nouvelles catégories de publics, les intentions proclamées tiennent souvent lieu de preuves. Or, l'intention si louable soit-elle, n'empêche pas de poser aussi la question de l'efficacité. Il serait hâtif de croire que la bonne volonté et les intentions proclamées suffisent à considérer que le projet, aussi généreux soit-il, est accompli.

Le manque d'imagination, la répétition de formes traditionnelles prétendument ludiques, le recours à des dispositifs abstraits contribuent, dans certains cas, et à diffuser une image peu dynamique de la médiation, et à et expliquer en partie la permanence des difficultés de certaines fractions des publics à s'approprier le contenu des expositions.

Dans les faits, il est difficile d'évaluer les effets de la médiation car si les buts sont toujours généreux et très ambitieux, ils demeurent le plus souvent vagues et irréalistes. La première tâche d'une recherche en ce domaine est de faire apparaître par le témoignage des acteurs et l'observation de séances d'intervention des médiateurs auprès des publics cibles, leurs objectifs, c'est-à-dire les changements culturels qu'ils souhaitent provoquer chez les visiteurs.

\subsection{Les objectifs d'une action de médiation : le cas des jeunes enfants}

Pour mesurer les effets des séquences de médiation conçues à l'intention d'enfants non lecteurs, la recherche a d'abord mis au jour les objectifs que l'équipe de médiateurs/concepteurs ont choisis, au moins implicitement, pour cette exposition. Pour cela, chaque animateur a été interrogé individuellement. Pourtant, les objectifs que chacun avait en tête, en créant et en animant l'exposition pour les 
enfants, étaient proches et nous avons pu établir sans difficulté la liste des objectifs visés.

On peut regrouper ces derniers en deux grandes catégories : les objectifs cognitifs qui en l'occurrence relèvent de ce que l'on appelle l'acquisition d'un sens esthétique, d'une part, et des objectifs plus nettement socio-affectifs, d'autre part. Parmi les premiers, on peut citer : reconnaître un tableau de Charles Camoin, percevoir le point de vue choisi par l'artiste pour peindre un tableau, apprécier les couleurs et le rôle de la lumière, développer l'observation pour percevoir réellement le travail d'un artiste, au-delà, gô̂ter la peinture de Charles Camoin.

Mais les animateurs voulaient aussi que les activités proposées autour des œuvres de ce peintre figuratif soient l'occasion d'un autre type d'apprentissage'. L'idée est que leur jeune public ressente et assume la fragilité et le caractère unique de ce patrimoine. De même, ils souhaitent que les activités soient aussi plaisantes et amusantes que possible afin de motiver plus tard d'autres visites de musées. Enfin, qu'ils apprennent à se comporter dans le lieu public-musée.

C'est la nature des perspectives de cette médiation qui a permis ensuite de construire un dispositif d'évaluation adapté. Dans ce cas, l'évaluation a utilisé un questionnaire administré individuellement à chaque enfant. Les questions ont été adaptées à des enfants de quatre à six ans par le choix rigoureusement contrôlé des mots et le recours, chaque fois que cela était possible, à des items visuels. Le questionnaire a été testé à plusieurs reprises pour vérifier qu'il soit compris et opérationnel. Les questions visaient à explorer différents thèmes abordés, tous en rapport avec les objectifs recensés.

\subsection{Le déroulement d'une activité de médiation .}

Pour atteindre ces objectifs et pour que la découverte des enfants soit la meilleure possible pour eux, les concepteurs/ animateurs de l'exposition ont avant tout choisi que la rencontre avec la peinture de Charles Camoin se fasse au cours d'un parcours interactif et ludique à travers lequel les enfants apprennent à écouter, à observer, à partager avec autrui. Ce moment au Préau des Accoules doit être unique, car c'est leur première rencontre avec une institution muséale.

1 Les tableaux exposés à cette occasion montrent des paysages, un port, des scènes de la vie quotidienne avec des jeux de couleurs et de lumière bien marqués. 
Pour ce faire, l'animation se décompose en plusieurs temps. Avant tout, dès que les enfants sont accueillis dans le Préau des Accoules, l'animateur qui a en charge la classe ou le groupe, explique le déroulement des activités qu'ils vont effectuer. Tout en les faisant participer, par un système de questions/réponses, les enfants sont informés de ce qu'est un musée, de ce qu'ils peuvent y découvrir et à quoi cela sert. Ils apprennent aussi que le Préau des Accoules est un musée spécialement conçu pour eux et qu'ils pourront profiter pleinement de ce lieu et le découvrir à leur aise, sans qu'aucun autre groupe ne vienne les déranger.

Les animateurs expliquent aussi aux enfants que si le Préau des Accoules, comme les autres musées, possède des objets uniques, les enfants vont devoir faire attention et respecter certaines règles, afin que d'autres visiteurs puissent eux aussi profiter de ces nombreux trésors. Il s'agit là en fait, tout en leur expliquant leurs droits et leurs devoirs et le pourquoi de ceux-ci, de les responsabiliser.

$\mathrm{Au}$ cours de ces quelques échanges, les règles de l'animation, elles-mêmes, sont évoquées :

Alors je vais vous poser une question. Quand je vous pose une question comme ça, vous levez le bras, puis vous pouvez répondre. On ne parle pas tous en même temps parce que sinon on ne s'entend plus !... Sinon on perd beaucoup de temps à faire de la discipline, c'est ça de moins pour jouer d'accord?

Après cette mise au point l'animation commence. La première partie permet aux enfants de découvrir, avant qu'ils ne voient les quatre toiles sélectionnées, Camoin et son univers. Un premier jeu, par un système de palette et de différentes plaquettes de couleurs, propose aux enfants à distinguer les nuances entre valeurs claires et valeurs foncées. L'enfant découvre avec étonnement qu'il existe un rouge différent de celui qu'il a sur ses chaussettes... C'est une première étape.

Un second jeu développe cette première notion. Les enfants placés face à une porte fenêtre fictive doivent imaginer être chez Charles Camoin. C'est la nuit, puis le jour se lève. Cette animation lumineuse se prolonge afin de montrer les différents moments de la journée jusqu'au retour de la nuit. Puis, en demandant aux enfants de fixer une couleur, l'animateur recrée et simule les différents moments d'une journée. Les enfants peuvent alors distinguer les variations que 
subissent les couleurs en fonction de la quantité de lumière au cours de la journée.

En plaçant l'un des enfants dans la porte fenêtre, les autres jeunes visiteurs peuvent appréhender la relation entre l'ombre et la lumière... Ces deux jeux abordent des notions que les enfants vont retrouver dans les quatre toiles du peintre. Ils ont été imaginés pour leur apporter une aide efficace pour observer les tableaux choisis. Lorsque les enfants ont bien compris de quoi il s'agit, ils sont alors prêts à les observer. C'est la seconde partie de l'animation. Comme pour les jeux, l'animateur, par un jeu de questions/réponses, tente de leur faire retrouver les notions présentées auparavant. Les enfants doivent indiquer sans erreur à quels moments de la journée Camoin a peint chacune des toiles que l'animateur désigne. Puis, rechercher le point commun qui existe entre elles. Ces deux étapes sont relativement rapides : 30 à 45 minutes. Ce temps limité et le petit nombre de tableaux contribuent à ce que l'enfant ne sature pas à cause d'un nombre trop important d'informations et qu'il ne ressente une contrainte due à la longueur de l'activité.

La troisième partie de l'animation est réservée à des jeux qui permettent de rappeler, de manière ludique, les connaissances présentées précédemment. Chaque tableau fait l'objet de plusieurs jeux disposés à proximité des œuvres du peintre. Après la présentation des différentes activités par l'animateur, les enfants peuvent les découvrir et jouer à leur rythme. Ces derniers, qui jusque là ont été très encadrés, doivent alors apprendre à ne pas se bousculer et à ne pas se disputer mais surtout à attendre leur tour, afin de faire leur propre découverte, comme à céder leur place une fois qu'ils ont terminé comme leur a expliqué l'animateur. Durant cette période (elle dure environ trois quarts d'heure), les adultes interviennent aussi peu que possible et les enfants apprennent à visiter ensemble.

\subsection{Les résultats de l'évaluation}

Pour pouvoir mesurer si tous les objectifs ont été atteints, le questionnaire a été administré à des enfants de quatre écoles maternelles et d'un centre aéré qui ont accepté de participer à cette évaluation. Ces jeunes visiteurs, qui avaient sensiblement le même âge (entre 4 et 6 ans) et qui appartenaient à des milieux sociaux différents, ont été interrogés individuellement à trois reprises le jour de leur visite, une semaine plus tard et un mois après leur passage au Préau 
des Accoules. Ceci afin de vérifier si les réponses des enfants dépendent (ou pas) de l'effet de récence de leur activité. Au total, 189 questionnaires complétés ont pu être utilisés.

Nous avons vu qu'avec l'exposition Charles Camoin, l'équipe du musée a tenté d'apprendre aux enfants à établir un contact visuel soutenu avec les œuvres, à repérer les éléments importants, à organiser les informations pour déchiffrer un tableau, tout en lui procurant du plaisir. Pour évaluer si par exemple cette habileté avait été acquise, une série de reproductions de six toiles leur a été présentée, dont les quatre peintures de Charles Camoin (exposées au Préau des Accoules) et deux distracteurs : un autre tableau de Camoin, Fenêtre ouverte à Cannes, 1956, reprenant certains détails des toiles du Préau et un tableau, La plage de Hove avec les bateaux de pêche, de Constable (artiste anglais du XIX ${ }^{\mathrm{e}}$ siècle).

Lorsqu'une des reproductions est reconnue comme étant une des toiles de l'exposition Camoin, on propose aussi à l'enfant de répondre à trois questions supplémentaires concernant des détails du tableau et cela sans qu'il dispose de la reproduction de l'œuvre.

Les résultats montrent que les quatre toiles de Camoin sont presque toujours reconnues : $99 \%$ pour le tableau Lola à l'ombrelle jaune, $95 \%$ pour la Fenêtre de l'atelier à Saint-Tropez, $94 \%$ pour L'étoile Vesper à la fenêtre de la salle à manger et $89 \%$ pour Le port au voilier vert. En ce qui concerne les deux distracteurs, les pourcentages indiquent que la marine de Constable a été bien distinguée comme ne faisant pas partie de l'exposition à $87 \%$. Par contre, celle de Camoin n'a pas été clairement identifiée puisque $76 \%$ ont déclaré l'avoir vue au Préau des Accoules. Cette erreur est cependant intéressante car elle semble indiquer que les enfants ont, en quelque sorte, perçu le style de Camoin. Les enfants y reconnaissent des détails communs à d'autres tableaux de l'exposition comme la mer, la portefenêtre... Comme le remarque un enfant, devant ce distracteur intitulé Fenêtre ouverte à Cannes: "J'ai vu ce tableau au Préau, mais le fauteuil n'était pas à la même place".

Ces résultats montrent donc que les enfants ont été plutôt attentifs pendant leur visite et qu'ils sont capables, à l'issue de celle-ci, de réussir des épreuves de reconnaissance et de rappel. On peut donc supposer qu'un apprentissage a bien eu lieu.

Un détail est cependant à méditer quand on analyse les scores obtenus par chacun des tableaux lors des tests de reconnaissance ou de rappel. Nous avions pris la précaution de demander aux médiateurs 
quels étaient les tableaux de l'exposition qu'ils préféraient. Or, il y a une correspondance nette entre les réponses attendues des enfants et les préférences de chaque médiateur vis-à-vis des toiles de Camoin. Cette relation montre toute l'importance de l'influence de l'animateur dans cette forme de médiation sur la qualité des observations faites par les enfants.

On a, par ailleurs, cherché à savoir si les enfants avaient pris du plaisir et s'étaient amusés pendant la visite et dans l'ensemble ils ont répondu par l'affirmative : 90,5\% des enfants affirment s'être amusés pendant l'animation, $6,3 \%$ ont dit qu'ils se sont ennuyés. Ce score est habituel pour ce type d'enquête et il ne faut pas lui accorder trop d'importance. On peut cependant supposer que cette condition a servi de facteur de stimulation pour la découverte par les enfants de la peinture de Camoin, des couleurs, des variations de lumière et pour la mémorisation de certains détails des toiles exhibées.

Il semble que tout en ayant pris du plaisir les enfants se sont familiarisés avec la peinture et, en particulier, avec celle de Charles Camoin. Il reste à déterminer si l'apprentissage des règles de comportement a connu le même succès.

\section{La médiation culturelle comme forme de régulation sociale}

On a souvent tourné en dérision le guide de musée qui débite sempiternellement le même discours stéréotypé à l'adresse de chaque groupe de visiteurs. Le moderne accompagnateur, comme le docte conférencier, et en fait tout animateur ou médiateur, qui s'interpose entre le public et l'offre culturelle, n'a sans doute plus grand-chose de commun avec ce stéréotype. Pourtant, il est évident que toute médiation proposée au public matérialise et condense la volonté de l'institution de conseiller un type de visite, voire de suggérer un faire voir et, au-delà, de solidariser l'offre culturelle et son mode d'usage. En somme, tout équipement muséographique serait, non seulement dépositaire d'un fonds culturel à conserver, mais aussi le seul habilité à conduire le travail de communication et de médiation des expositions.

Le contrôle du "droit de parole" dans l'exposition d'art (par exemple, au musée du Louvre ou à Versailles) et dans les monuments historiques matérialise le souci de la profession de ne pas laisser 
proférer n'importe quel commentaire sur les œuvres et le patrimoine'. Seule l'institution est supposée détenir le vrai (ou le bon ?) discours d'interprétation à l'intention des visiteurs.

L'absence physique d'un guide ou d'un animateur n'est pas un gage de plus grande liberté. En intégrant les aides à la visite (signalétique, étiquettes, feuillets, audioguides, etc.) dans les équipements muséographiques, les professionnels de la médiation tentent, en quelque sorte en amont, de créer des conditions favorables à une appropriation de l'exposition et de ses contenus par les visiteurs de façon autonome. Mais il est clair que cette autonomie est comme pilotée par les orientations choisies et retenues par ceux qui ont conçu ces dispositifs d'interprétation.

Comme le souligne ce responsable d'un service culturel d'un musée d'art :

Lorsque dans une exposition, le visiteur n'a aucune difficulté à rattacher les œuvres à un concept général, qu'il ne se rend même pas compte que tout cela est pensé dès le départ, que les moyens sont intégrés, c'est pour moi quelque chose de réussi.

Tous ces outils, mis à la disposition du visiteur, qu'il soit élève ou adulte, l'incitent effectivement à adopter une attitude active de visite mais cette autonomie est comme contrôlée par les moyens proposés tout au long de son parcours dans l'exposition.

\subsection{Le point de vue des acteurs de la médiation culturelle dans le musée}

Dans les témoignages recueillis auprès des médiateurs revient un leitmotiv: le musée ne peut devenir une ressource culturelle ou éducative qu'à la condition qu'on en connaisse le "mode d'emploi". Au même titre que d'autres lieux-ressources d'enrichissement de la culture et des connaissances, comme les bibliothèques, des moyens et des méthodes d'accès à la richesse et au potentiel du média exposition et du musée en général, doivent être suggérés voire même enseignés aux utilisateurs. Du coup, on assigne, à la médiation culturelle mise en œuvre dans les musées, un programme complémentaire de la seule

1 Il est interdit dans les musées nationaux de commenter le patrimoine à haute voix à l'intention d'un groupe de visiteurs. Sauf à posséder le titre de guide-conférencier et de demander ensuite l'autorisation de le faire au directeur de l'institution. 
valorisation culturelle de la collection ou du donné à voir : permettre aux différentes catégories de visiteurs d'accéder, de façon autonome, aux ressources muséales ou expographiques pour en retirer la vision optimale.

En somme, que la médiation soit prise en charge par un animateur ou qu'elle soit induite (de façon implicite) dans la mise en exposition, il n'en demeure pas moins que les objectifs sont les mêmes : d'abord donner du sens (et même un sens) aux thématiques et aux contenus abordés par l'exposition, mais aussi susciter l'intérêt et la motivation pour la ressource muséale en général, tout en incitant le public à acquérir les "bonnes" méthodes de visite.

Je me préoccupe beaucoup du quotidien de l'enfant. Le jeune qui entre dans le musée, c'est ma priorité. Il faut que le jeune qui est entré ce matin, ressorte du musée avec une expérience de qualité. Qu'ils partent d'ici avec une vision du musée, avec une perception plus ouverte qui leur donne le goût de revenir. Pour moi, cela est très important, car c'est le public de demain [responsable des visites dans un musée d'art contemporain].

Les enfants retiennent beaucoup d'informations, sauf que si cela ne s'inscrit pas selon une motivation, ils ont de la difficulté à donner un sens à l'histoire. C'est ici, à mon avis, c'est dans les musées qu'on peut sensibiliser les enfants au patrimoine et à la conservation de ce patrimoine [responsable des programmes éducatifs dans un musée d'histoire et d'archéologie].

Tout projet d'acculturation, au sens large, des différentes catégories de public inclut certes un travail centré sur le contenu culturel à transmettre, mais aussi la volonté de sensibiliser les visiteurs aux valeurs culturelles du musée. Cameron ${ }^{1}$ a montré clairement que les professionnels des musées ont aussi la responsabilité d'initier les visiteurs à l'usage du musée. Ils doivent, suggère-t-il, enseigner le langage et les méthodes d'utilisation des ressources [éducatives] muséales. Au visiteur d'apprendre à se servir du musée comme une ressource pour initier, approfondir ou

\footnotetext{
${ }^{1}$ D. Cameron, "Un point de vue : le musée considéré comme un système de communication et les implications de ce système dans les programmes éducatifs muséaux, in Vagues: une anthologie de la nouvelle muséologie (traduit de l'anglais), Savigny-le-Temple, MNES, 1968, pp. 259-270.
} 
renouveler ses connaissances, à partir des dispositifs de médiation mis en œuvre par les professionnels.

Le travail avec les gens c'est une forme d'éducation. Je soulignerais même le fait que ce n'est pas juste de donner de l'information au visiteur. Lorsque l'on reçoit des gens dans le musée, on essaie de les sensibiliser au fait qu'ils sont dans un lieu public, un lieu où on conserve des choses, où il y a des règles. On n'est pas au terrain de jeu. On n'est pas dans la cour de récréation. C'est une visée éducative qui est large. [agent des programmes éducatifs dans un équipement muséographique axé sur les sciences naturelles].

Cette volonté de guider aussi les pratiques de visite et de suggérer au public un mode opératoire peuvent faire l'objet de deux lectures différentes. Ou bien, elle montre que l'institution muséale fait preuve d'une réelle volonté de démocratiser et de rendre souple et accessible la fréquentation du musée. En dévoilant le mode d'emploi des expositions, elle permet à d'autres groupes que le seul public averti et connaisseur d'oser visiter ces lieux austères et intimidants que sont les musées. Ou bien, ces nouvelles formes d'intervention culturelle, sous prétexte d'offrir la possibilité à tous d'apprendre à mieux découvrir le musée, cherchent à imposer un ensemble de règles qui disciplinent (au sens de Foucault ${ }^{1}$ ) la visite.

\subsection{Le cas du jeune public}

Dans le cas de l'action auprès du jeune public que nous avons déjà évoquée, l'affirmation de la nécessité pour les médiateurs de viser aussi des objectifs socio-affectifs est évidemment affirmée avec netteté. Pour évaluer ce que ces nouvelles formes de médiation peuvent apporter de différent, par rapport à l'enseignement académique, on a cherché à apprécier non seulement les effets de la découverte guidée d'œuvres mais aussi, les effets vis-à-vis de la reconnaissance du lieu musée et de l'inculcation des comportements adéquats comme des règles de conduite à adopter dans cette institution.

Depuis Durkheim ${ }^{2}$, on sait que la mission première de l'école vis-à-vis des enfants âgés de 4 à 6 ans est de leur apprendre à vivre dans la société. En visitant un musée, les jeunes enfants s'éloignent de

1 M. Foucault, Surveiller et punir. Naissance de la prison, Paris, Gallimard, 1975.

2 E. DURKheIM, Éducation et sociologie, Paris, PUF, 1993 (1922). 
leurs deux cadres de référence habituels : la cellule familiale et l'école. Se rendre au Préau des Accoules les conduit à une triple découverte : évoluer dans un lieu dont la plupart d'entre eux n'ont pas l'habitude, travailler avec des adultes qu'ils ne connaissent pas (les animateurs), adopter une posture de découverte vis-à-vis de la tâche proposée et tout cela en interaction avec les autres enfants.

En effet, en même temps que les jeunes visiteurs découvrent la peinture de Charles Camoin, ils apprennent ce qu'est un musée, à quoi il sert et quelles sont les règles qu'il est nécessaire de respecter dans ce lieu, par rapport aux objets qu'il contient, aux personnes qui y travaillent, et aussi par rapport à leurs camarades qui le visitent en même temps qu'eux.

Il est clair que la médiation assure son rôle tant sur le plan cognitif que sur le plan socio-affectif : la présence des adultes est permanente du début à la fin de cette activité, conduite de façon collective, ordonnée et structurée entre déplacements, moments d'activités autour d'une œuvre et enfin période plus ludique et plus souple mais visant à conforter les acquisitions précédentes.

Tout au long de chaque séance, l'adulte assure son rôle de médiation esthétique tout en effectuant un contrôle de la dynamique relationnelle et groupale. $\mathrm{Il}$ rappelle à l'ordre, attire l'attention des étourdis, avertit, insiste et admoneste au besoin. En effet, les animateurs interviennent lorsque les enfants se disputent ou ne respectent pas les règles du Préau des Accoules et surtout anticipent avec enthousiasme. Généralement, les enfants qui découvrent le Préau des Accoules s'entraînent mutuellement de découverte en découverte : "Oh! Viens voir ce jeu !" et sont impatients d'aller de l'avant.

Notre enquête d'évaluation a cherché à contrôler deux paramètres différents. Les enfants à l'issue de leur visite sont-ils tout d'abord capables de différencier le musée d'autres lieux publics ? Nous avons cherché à le savoir à l'aide d'une épreuve de reconnaissance qui comportait une série de six photographies représentant différents espaces (supermarché, jardin public, salle de jeu, musée, classe et Préau des Accoules). Une condition élémentaire de régulation de la conduite sociale est en effet de repérer et distinguer le lieu dans lequel on se trouve pour adopter le comportement adéquat.

A-t-on ou n'a-t-on pas le droit d'agir de telle ou telle façon dans un musée ? Pour le second volet, nous avons posé aux enfants une série de questions très simples formulées avec leurs mots sur ce que les autres enfants peuvent faire au Préau des Accoules. Il s'agit ici 
d'explorer la conscience que le jeune visiteur a de la conduite à tenir dans ce type de lieu public.

Sans surprise, la grande majorité des enfants $(96 \%)$ reconnaît sans difficulté le Préau des Accoules parmi les autres photos d'espaces publics. Identifier le lieu où l'on vient de passer du temps n'est pas en soi difficile. Mais ce sont les autres réponses, celles qui sont donc erronées qui sont ici importantes (les enfants pouvaient retenir plusieurs photographies d'espaces publics).

Ainsi, $41 \%$ des enfants choisissent aussi la photographie du musée des Beaux-Arts de Marseille lorsqu'elle leur est montrée. Les enfants semblent donc traiter certaines ressemblances entre ces deux lieux : la présence d'un parquet, de murs peints en blanc et surtout, comme pour l'exposition qu'ils ont vue, l'existence de tableaux accrochés aux murs. Cette identification erronée est l'indice d'une perception plutôt judicieuse.

Les autres choix des enfants sont également fort instructifs. $23 \%$ des enfants ont confondu le Préau des Accoules avec une salle de classe et une salle de jeu. On peut se demander si ces deux lieux n'ont pas été rapprochés puisque les enfants les fréquentent à l'occasion d'activités pédagogiques et éducatives dans lesquelles ils sont encadrés par leur maîtres, un peu comme lors de leur visite au Préau où ces derniers, sans intervenir, les accompagnent malgré tout. Ces enfants assimileraient à l'école tous les espaces qu'ils fréquentent avec leurs maitres. Les autres erreurs ( $21 \%$ des enfants ont confondu le Préau avec un supermarché et $16 \%$ avec un jardin public) sont peut-être plus anecdotiques.

Pour ce qui est de la conduite dans le musée, les réponses indiquent que les enfants discriminent sans ambiguilté les énoncés correspondant à ce qui est autorisé ou interdit au Préau des Accoules. $91,5 \%$ des enfants répondent que l'on ne peut pas y dormir, $82,5 \%$ que l'on ne peut pas y manger, et surtout $94,7 \%$ que l'on n'a pas le droit de courir dans les salles...

Réciproquement, ils citent sans erreur, dans leur grande majorité, ce que l'on peut effectivement faire dans un musée. Ainsi, $86,8 \%$ des enfants affirment que l'on peut apprendre des choses en relation avec la lumière et les couleurs et $66,7 \%$ pensent que l'on peut travailler dans un tel lieu. $94 \%$ des enfants répondent qu'au musée, on a le droit de voir des tableaux et $83 \%$ que l'on n'a pourtant pas le droit de les toucher. Évidemment, d'autres fonctions du musée, comme la conservation des œuvres, présentent plus de difficulté de compréhen- 
sion pour les enfants de cet âge. Ainsi, $63 \%$ ont affirmé que l'on peut conserver de vieilles choses au Préau des Accoules.

Pourtant, si la plupart des comportements semblent avoir été acquis à l'école ou dans un autre cadre, d'autres actions n'ont pu faire l'objet d'apprentissage que dans une institution muséale ou encore au Préau des Accoules. Plus encore, les enfants semblent avoir intégré les règles au point d'estimer avec finesse les transgressions tolérables. En effet, $97,3^{\circ} \%$ d'entre eux ont répondu qu'ils ne peuvent pas grimper sur les colonnes, mais $87,8^{\circ} \%$ pensent qu'ils ont le droit de s'amuser dans un tel lieu. Un enfant a même introduit une nuance au verbe s'amuser en répondant: Oui, on peut s'amuser, mais on ne peut pas faire la foire!

Les enfants semblent donc avoir assimilé ce que les enseignants et les animateurs attendent d'eux. Il est possible qu'au fur et à mesure de la séance d'animation, l'enfant ait analysé ce nouvel espace et que son comportement se soit affiné en fonction de cette expérience qu'il traite en somme dans la continuité des règles de l'école où l'interdit n'a de sens qu'en rapport avec sa possible transgression.

Ceci semble aussi se vérifier par les nuances que les enfants introduisent dans ce qu'ils prétendent pouvoir faire au travers, d'une part, les réponses qu'ils formulent aux questions qui leur sont posées par l'adulte-enquêtrice, et d'autre part, la réalité de ce qu'ils font réellement dans cette situation. Ainsi, s'ils ont répondu de manière conforme aux attentes du personnel du musée, une observation de la visite montre évidemment qu'ils ne respectent pas tous physiquement et à chaque instant ces règles. En effet, ils ne se privent pas de courir et de grimper dès que l'occasion s'en présente, quitte à ce que leurs attitudes soient ainsi en désaccord avec leurs affirmations lors de l'entretien. En même temps que les enfants ont appris ce que le musée attendait d'eux, ils ont également apprécié leurs marges de latitude par rapport à cette "norme"1.

La plupart des enfants qui ne se sentent pas observés, au cours des activités ludico-éducatives mises en place par l'équipe du musée, courent dans le Préau des Accoules ou encore taquinent leurs camarades. Ils ont à ce point compris les règles imposées qu'ils peuvent s'accorder un certain degré de liberté par rapport à ces consignes sans pourtant les ignorer.

1 I. MARTINEZ et A. VASQUEZ-BRONFMAN, La socialisation à l'école, une approche ethnographique, Paris, PUF, coll. "L'éducateur", 1996, p. 204. 


\section{La médiation : projet culturel ou régulation sociale du 'bon" goût?}

Poulot $^{1}$, évoquant la naissance tumultueuse des musées à la fin du XVIIre siècle, montre comment le projet démocratique de faire accéder le peuple aux trésors de la culture s'est très vite heurté aux dangers et aux menaces que cette ouverture risquait de faire courir aux collections rares et précieuses exposées dans les lieux autrefois réservés à la noblesse ou au clergé. Et il souligne comment la création d'un corps de gardiens (revêtus d'un uniforme proche de celui des gens d'armes) visait, non seulement à protéger et surveiller les chefs d'œuvres, mais aussi à imposer le seul usage culturel dans les espaces ou palais que la population utilisa d'emblée, aussi bien à des fins culturelles, que pour s'abriter, se nourrir, fumer, se reposer, s'interpeler et deviser à haute voix avec les autres. Cette évocation de la naissance du musée prête à sourire tant le raffinement ou la seule bienséance des civilisations contemporaines nous ont fait perdre de vue une partie de ce que recouvrent l'apprentissage systématique et la lente intériorisation de nouveaux usages.

Le temps des gardiens qui encadrent et disciplinent les foules estil aujourd'hui dépassé ? Comment comprendre la fermeture récente du centre Georges Pompidou à Paris sans la mettre en relation avec son succès paradoxal ? On sait que cet équipement, à l'architecture si contemporaine qu'elle a heurté bien des esprits lors de son ouverture, est entre autres un musée d'art moderne et un lieu d'exposition de l'art contemporain installé au centre de Paris par la volonté d'un ancien Président de la République Française. Or, alors que la fréquentation du musée est demeurée modeste, le centre à été étouffé et usé prématurément par un immense public de badauds, de promeneurs qui déambulait dans les espaces laissés en libre accès tandis que le vaste forum du rez-de-chaussée était utilisé comme lieu de rencontre et de discussion par des groupes d'immigrés bienheureux d'avoir trouvé du travail.

La réussite des politiques culturelles, quelles qu'elles soient, dépend évidemment des acteurs qui sont chargés de les mettre en œuvre. Les conservateurs et les gardiens étaient donc les deux figures tutélaires du musée classique. Quelles sont celles qui leur ont succédé

1 D. Poulot, "Bilan et perspectives pour une histoire naturelle des musées, Publics et Musées, n 2, 1992, pp. 125-147. 
dans un équipement muséographique contemporain ? La figure du conservateur s'est brouillée : on lui oppose celle du manager, du concepteur d'exposition. Le gardien n'a pas disparu mais tout ce qui est ostentatoire chez le moderne agent de surveillance ou d'accueil du public a été comme effacé. On le reconnaît à son badge ou au fait qu'il soit toujours assis. Il est discret et cherche en somme à se faire oublier. On lui a retiré le droit de parole : il n'a pas compétence à commenter le donné à voir puisque les guides, animateurs et médiateurs sont précisément apparus pour le faire. Certes, il surveille et symbolise, même s'il le fait discrètement, le respect des règles et de l'ordre assurant par la présence des cadres de l'expérience et du rituel (au sens de Goffman) de la visite admirative ; mais qui donc assume la charge du "bon" faire culturel et des "vrais" modes d'usage des expositions?

Revenons à la médiation qu'elle soit active (en face à face) ou proactive (la conception d'aides à la visite). Nous avons relevé que les politiques de médiation destinées au public scolaire comme aux adultes, ont aussi pour but d'inciter à acquérir de l'autonomie dans la visite de l'exposition. L'usage de ces aides à la visite est donc également destiné à apprendre le mode d'emploi de l'exposition. Ils constituent des dispositifs pédagogiques à double visée. Les professionnels insistent en effet sur la qualité d'une médiation qui s'applique aussi à susciter des attitudes et des comportements destinés à faire "apprendre à apprendre" ce que l'on peut retirer de la ressource muséale.

De même, certains des résultats de l'étude conduite auprès des enfants non lecteurs sont importants pour poursuivre notre réflexion. Les enfants se sont familiarisés avec la peinture et en particulier avec les œuvres et le style du peintre Camoin. Ils ont développé leur sens de l'observation surtout vis-à-vis des variations de valeurs et de couleurs en fonction de la lumière des différents moments d'une journée. Simultanément, les médiateurs se sont efforcé de leur faire assimiler la conduite à tenir dans un lieu public différent du cadre scolaire.

Des pourcentages de résultats -convergents avec les intentionsaussi élevés que ceux enregistrés dans notre enquête semblent indiquer que la grande majorité des enfants a commencé à apprendre comment se comporter dans un musée, alors que, pour la plupart d'entre eux, le Préau des Accoules est une première expérience muséale. Ces résultats semblent montrer que, tout en s'amusant, ils 
ont appris non seulement quelques rudiments de culture esthétique mais aussi à se comporter face à un lieu et des adultes nouveaux. Ils ont ainsi étendu leurs apprentissages des conduites à tenir dans la société ${ }^{\text {. }}$.

Il est clair que nos deux petites enquêtes établissent que la médiation a un projet plus ambitieux que la seule optimisation culturelle d'une visite : faire acquérir une sorte d'habitus muséal c'est-àdire un :

système de dispositions durables et transposables, structure structurée prédisposée à fonctionner comme une structure structurante, c'est-à-dire en tant que principe générateur et organisateur de pratiques et de représentations qui peuvent être objectivement adaptées à leur but sans supposer la visée consciente des fins et de la maîtrise expresse des opérations nécessaires pour les atteindre $(. . .)^{2}$.

Les enfants ont, semble-t-il, instinctivement transposé les règles apprises, probablement à l'école ou ailleurs, au cas du musée (sans probablement en avoir conscience). Le musée se range peut-être de la sorte plus facilement dans la continuité de l'école dont très vite on a noté qu'elle n'a pas pour seule fonction de transmettre des savoirs.

On envoie tout d'abord les enfants à l'école, non pour qu'ils y apprennent quelque chose, mais pour qu'ils s'y accoutument à rester tranquillement assis et à observer ponctuellement ce qu'on leur ordonne, afin que dans la suite ils sachent tirer parti de toutes les idées qui leur viendront ${ }^{3}$.

Et en effet, l'école maternelle et les autres institutions de la petite enfance (jardin d'enfants, halte-garderie...) ont pour finalité première, affirmait déjà Durkheim au début du siècle, de socialiser l'enfant, de lui faire intégrer les règles premières de la vie collective. Pour ce

1 Soulignons cependant une ambiguiité. Ces conduites, adoptées par la grande majorité des enfants, alors que la plupart ne sont jamais allés dans un musée, ne sont-elles pas la continuation des attitudes imposées par l'école ? En effet, l'école maternelle est souvent la seule structure collective que connaissent les enfants de 4 à 6 ans. Il est possible que les enfants, accompagnés par leurs maîtres au Préau des Accoules, aient adopté, face à une nouvelle situation, dans un contexte particulier, certaines conduites apprises à l'école.

2 P. BouRDIEU, "Les trois états du capital culturel", Actes de la recherche en sciences sociales, 1979, pp. 3-6.

3 E. KANT, Réflexions sur l'éducation, Paris, Vrin, 1966, p. 36. 
faire, elle leur inculque, comme l'a montré Grignon ${ }^{1}$ pour l'enseignement professionnel, non seulement les usages pertinents des espaces et des lieux : espace de jeu (la cour de récréation, le bac à sable), espace de travail (le coin peinture, les activités graphiques), espace de vie (les toilettes, la salle de repos, la cantine, le lieu où l'on accroche ses vêtements...) mais aussi des temps, de l'horaire, des périodes qui rythment le déroulement journalier (repos, travail, jeu, repas, activité libre ou dirigée...). Ce découpage et ce contrôle, en apparence anodins du temps et de l'espace, ont pour effet de simultanément créer des catégories et de les hiérarchiser tout en les ancrant sous la forme de modèles de conduites auxquels l'enfant doit se plier dans le quotidien. Ils permettent à l'institution d'imposer puis de maintenir un fonctionnement harmonieux au sein de l'institution qui de la sorte préfigure l'ordre social.

Les acteurs de la médiation que nous avons rencontrés, aussi bien quand on les observe et qu'on évalue certains des effets de leur travail que lorsqu'on les interroge sur leur buts et leurs conceptions de leurs tâches, manifestent donc concrètement qu'ils interviennent au delà de l'acquisition d'une culture thématique ou du sens esthétique. Le travail de médiation opère une forme de régulation sur deux plans différents. D'abord, le travail de médiation vise, par ses objectifs d'acculturation à agir sur le beau et la valeur des perceptions ou des émotions esthétiques. En cela il est déjà un régulateur social en ce qu'il confirme et soutient les choix des directeurs des musées et des équipements muséographiques qui fixent et délimitent ce qui est digne d'être contemplé et admiré. Ainsi, les médiateurs contribuent à leur façon à établir ce qui constitue le bon goût, celui des vrais "connaisseurs"2.

Pour les nouveaux visiteurs et particulièrement les jeunes enfants, la médiation se donne aussi pour tâche, comme l'assure l'éducation, de les socialiser (au sens de Dubar ${ }^{3}$ ). On peut, en effet, appliquer sans difficulté au musée la grille d'interprétation des fonctions sociologiques de l'école décrite par Dubet et Martucelli

1 C. GRIGNON, L'ordre des choses; les fonctions sociales de l'enseignement technique, Paris, Éd, de Minuit, 1971.

2 F. HASKELL, La norme et le caprice, Paris, Flammarion, 1993.

3 C. DuBAr, La socialisation : construction des identités sociales et professionnelles, Paris, Colin, 1991.

4 F. Dubet et D. Martuceldi, A l'école : sociologie de l'expérience scolaire, Paris, Éd. du Seuil, 1996. 
Chacun, selon le modèle de référence retenu, pourra prêter ainsi à la médiation muséale, soit une contribution à la fonction de reproduction des inégalités entre des catégories sociales, soit encore une place dans le système complexe des régulations entre générations ou catégories d'acteurs, soit enfin une marge d'autonomie et d'individuation au sein des comportements collectifs. 